УДК 517.55

\title{
Singular Points of Complex Algebraic Hypersurfaces
}

Irina A. Antipova*

Institute of Space and Information Technologies

Siberian Federal University

Kirensky, 26, Krasnoyarsk, 660074

Russia

Evgeny N. Mikhalkin ${ }^{\dagger}$

Avgust K. Tsikh ${ }^{\ddagger}$

Institute of Mathematics and Computer Science

Siberian Federal University

Svobodny, 79, Krasnoyarsk, 660041

Russia

Received 03.09.2018, received in revised form 22.10.2018, accepted 28.10.2018

We consider a complex hypersurface $V$ given by an algebraic equation in $k$ unknowns, where the set $A \subset \mathbb{Z}^{k}$ of monomial exponents is fixed, and all the coefficients are variable. In other words, we consider a family of hypersurfaces in $(\mathbb{C} \backslash 0)^{k}$ parametrized by its coefficients $a=\left(a_{\alpha}\right)_{\alpha \in A} \in \mathbb{C}^{A}$. We prove that when $A$ generates the lattice $\mathbb{Z}^{k}$ as a group, then over the set of regular points a in the A-discriminantal set, the singular points of $V$ admit a rational expression in a.

Keywords: singular point, A-discriminant, logarithmic Gauss map.

DOI: 10.17516/1997-1397-2018-11-6-670-679.

\section{Introduction}

By the general algebraic hypersurface (or the A-hypersurface) we mean the algebraic set $V$ defined by the equation in $k$ unknowns $y=\left(y_{1}, \ldots, y_{k}\right) \in(\mathbb{C} \backslash 0)^{k}$ :

$$
f\left(y_{1}, \ldots, y_{k}\right):=\sum_{\alpha=\left(\alpha_{1}, \ldots, \alpha_{k}\right) \in A} a_{\alpha} y_{1}^{\alpha_{1}} \ldots y_{k}^{\alpha_{k}}=0 .
$$

Here $A \subset \mathbb{Z}^{k}$ is a fixed finite set while all coefficients $a_{\alpha}$ are treated as independent variables. We assume that the set $A$ generates the lattice $\mathbb{Z}^{k}$ as a group. The set of polynomials (1) is identified with the space $\mathbb{C}^{A}$ of sequences $a=\left(a_{\alpha}\right)_{\alpha \in A}$ of dimension $N:=\# A$. We can think about $V$ as a family of hypersurfaces $V_{a}$ in $(\mathbb{C} \backslash 0)^{k}$ parametrized by coefficients $a \in \mathbb{C}^{A}$.

The aim of the present paper is to obtain explicit formulas for almost all singular points of the hypersurface $V$. Recall that a point $y \in V$ is said to be singular if the polynomial $f$ in (1) and all its partial derivatives $f_{y_{1}}^{\prime}, \ldots, f_{y_{k}}^{\prime}$ vanish at $y$. In the classical case, when $k=1$, the following formulas were given in $[1$, Ch.1, Th.1.5]: if the equation

$$
f(y):=a_{d} y^{d}+\ldots+a_{1} y+a_{0}=0
$$

*iantipova@sfu-kras.ru

${ }^{\dagger}$ mikhalkin@bk.ru

$\ddagger$ atsikh@sfu-kras.ru

(C) Siberian Federal University. All rights reserved 
has a unique multiple root $y=y(a)=y\left(a_{0}, \ldots, a_{d}\right)$, and its multiplicity equals two, then $y$ is given by rational expressions

$$
y=\frac{\Delta_{1}(a)}{\Delta_{0}(a)}=\frac{\Delta_{2}(a)}{\Delta_{1}(a)}=\ldots=\frac{\Delta_{d}(a)}{\Delta_{d-1}(a)}
$$

where $\Delta_{j}=\frac{\partial \Delta}{\partial a_{j}}$ are derivatives of the discriminant $\Delta(a)$ of the polynomial $f(y)$ in $(2)$. Analogous formulas for a unique root of multiplicity $\nu \geqslant 2$ are given in [5], where instead of using the discriminant $\Delta$, the resultant of $f$ and its derivative $f^{(\nu-1)}$ (with respect to $y$ ) of order $\nu-1$ is used.

We prove that almost all singular points $y(a)$ (actually, those that correspond to $a$ belonging to the regular part of the discriminantal set) admit a rational representation (Theorem 3 ). In the last section we consider an example with comments how the type of a singular point $y(a) \in V$ depends of the singular type of $a \in \nabla_{A}$.

\section{A-discriminant and the reduced equation}

Definition $1([1])$. Let $\nabla^{\circ}$ denote the set of all $\left(a_{\alpha}\right) \in \mathbb{C}^{A}$ such that the equation (1) has critical roots $y \in(\mathbb{C} \backslash 0)^{k}$, i.e. roots at which the gradient of $f$ vanishes:

$$
f(y)=\frac{\partial f}{\partial y_{1}}(y)=\ldots=\frac{\partial f}{\partial y_{k}}(y)=0 .
$$

The closure $\overline{\nabla^{\circ}}=: \nabla_{A}$ in $\mathbb{C}^{A}$ is said to be the A-discriminantal set.

In the set $\nabla_{A}$ is a hypersurface in $\mathbb{C}^{A}$, then by the $A$-discriminant one means an irreducible integral polynomial $\Delta_{A}$ in coefficients $a$ of $f \in \mathbb{C}^{A}$ which vanishes on $\nabla_{A}$.

The solution $y=y(a)$ to the equation $(1)$ is $(k+1)$-homogeneous (it satisfies $k+1$ homogeneity conditions), and the $A$-discriminant inherits this property. To see this, we consider the following action on the space $\mathbb{C}^{A}$ of polynomials (1). For $\lambda=\left(\lambda_{0}, \lambda_{1}, \ldots, \lambda_{k}\right) \in(\mathbb{C} \backslash 0)^{k+1}$ we define it as follows

$$
\lambda: f\left(y_{1}, \ldots, y_{k}\right) \rightarrow \lambda_{0} f\left(\lambda_{1} y_{1}, \ldots, \lambda_{k} y_{k}\right) .
$$

Observe that the set $\nabla_{A}$ is invariant under the $\lambda$-action. In terms of coefficients $\left(a_{\alpha}\right)$ of the polynomial $f$ this action can be written in the following form:

$$
a_{\alpha} \rightarrow \lambda_{0} \lambda_{1}^{\alpha_{1}} \ldots \lambda_{k}^{\alpha_{k}} a_{\alpha}, \quad \alpha \in A
$$

Here $\alpha_{1}, \ldots, \alpha_{k}$ are the coordinates of $\alpha$. In the toric part $(\mathbb{C} \backslash 0)^{A} \subset \mathbb{C}^{A}$ the orbits of this action are the equivalence classes with respect to the $(k+1)$-parametric subgroup defined by the immersion

$$
\left(\lambda_{0}, \lambda_{1}, \ldots, \lambda_{k}\right) \hookrightarrow \lambda_{0} \lambda_{1}^{\alpha_{1}} \ldots \lambda_{k}^{\alpha_{k}}, \quad \alpha \in A .
$$

Its injectivity follows from the fact that $A$ generates $\mathbb{Z}^{k}$. Renumerating the elements of $A$ as $\alpha^{1}, \ldots, \alpha^{N}$ we represent this immersion in the form

$$
\left(a_{\alpha}\right)=\lambda^{A},
$$


where $A$ is the matrix

$$
A=\left(\begin{array}{cccc}
1 & 1 & \ldots & 1 \\
\alpha_{11} & \alpha_{21} & \ldots & \alpha_{N 1} \\
\vdots & \vdots & \ddots & \vdots \\
\alpha_{1 k} & \alpha_{2 k} & \ldots & \alpha_{N k}
\end{array}\right)
$$

and

$$
\lambda^{A}=\left(\lambda^{a^{1}}, \ldots, \lambda^{a^{N}}\right)=\left(\lambda_{0} \lambda_{1}^{\alpha_{11}} \ldots \lambda_{k}^{\alpha_{1 k}}, \ldots, \lambda_{0} \lambda_{1}^{\alpha_{N 1}} \ldots \lambda_{k}^{\alpha_{N k}}\right)
$$

with $a^{j}$ being the columns of this matrix. Remark that we keep the notation $A$ (which was used for the set of exponents $\alpha$ in (1)) for this extended matrix. Thus, an equivalence class can be written in the form $\lambda^{A} \cdot g$ with the coordinate-wise multiplication. In order to parameterize all equivalence classes we represent them in the form of an $m$-parametric subgroup

$$
g=z^{C}, \quad z \in(\mathbb{C} \backslash 0)^{m},
$$

where $C$ is an $m \times N$-matrix with $m=N-k-1$. Choosing the matrix $C$ in such a way that the $N \times N$-matrix

$$
\widehat{A}=\left(\frac{A}{C}\right)
$$

is unimodular (with determinant \pm 1 ), we conclude that the transform

$$
\widehat{A}:(\lambda, z) \rightarrow \lambda^{A} \cdot z^{C}
$$

is an automorphism of the complex torus $(\mathbb{C} \backslash 0)^{A}$. Thus, for such $C$ the $m$-parametric subgroup $g=z^{C}$ parametrizes all equivalence classes modulo the subgroup $\lambda^{A}$. Denoting by $c^{\alpha}$ the column of the matrix $C$ indexed by an element $\alpha \in A$, we arrive at the following reduced equation for (1):

$$
f(y)=\sum_{\alpha \in A} z^{c^{\alpha}} y^{\alpha}=0,
$$

where the coefficients $z^{c^{\alpha}}=z_{1}^{c_{1}^{\alpha}} \ldots z_{m}^{c_{m}^{\alpha}}, \alpha \in A$, run over the $m$-parametric subgroup $z^{C}$ in $(\mathbb{C} \backslash 0)^{A}$. The discriminantal set of the equation (5) we denote by $\nabla_{A}^{\prime}$ and call it the reduced discriminantal set. The defining polynomial of $\nabla_{A}^{\prime}$ is obtained from the $A$-discriminantal polynomial $\Delta_{A}$. It is called the reduced discriminant.

By Kapranov's theorem [6] the reduced discriminantal set is birationally equivalent to the projective space $\mathbb{C P}^{m-1}$. Moreover, there is an explicit formula

$$
z=(B s)^{B}, \quad s \in \mathbb{C P}^{m-1},
$$

parametrizing $\nabla_{A}^{\prime}$. Clearly, then we get a parametrization of $\nabla_{A}$ as

$$
a=\left(a_{\alpha}\right)_{\alpha \in A}=\lambda^{A} \cdot(B s)^{B C} .
$$

\section{Parametrization of singular points}

The matrix $C$, extending $A$ in (4) defines a special matrix $B$, the so called Gale transform of $A$ (see [1, P. 225]). Namely, the inverse of the matrix $\widehat{A}$ can be represented in the following block form:

$$
(\widehat{A})^{-1}=(D \mid B)
$$


where $D$ and $B$ are blocks with $k+1$ and $m$ columns, respectively. They satisfy the relations

$$
A \cdot B=0, \quad A \cdot D=E_{k+1} .
$$

Remark that we can use columns $\alpha$ of $A$ to index the rows for $B$ writing them as $b_{\alpha}$. With the help of $B$ and $D$ we can formulate the theorem on singular points of the reduced hypersurface (5).

The most convenient reductions of the equation (1) are associated with matrices $C$ which contain $k+1$ zero columns at that the other $m$ columns form the unit matrix. Such matrices can be used for extension of $A$ to be unimodular if $A$ has $k+1$ columns, say $\alpha^{0}, \alpha^{1} \ldots, \alpha^{k}$, for which the columns

$$
\alpha^{1}-\alpha^{0}, \ldots, \alpha^{k}-\alpha^{0}
$$

form a unimodular $k \times k$-matrix $\delta$. In this case the reduction of (1) is just a fixation of the coefficients: $a_{\alpha^{0}}=a_{\alpha^{1}}=\ldots=a_{\alpha^{k}}=1$. We can use such a reduction when $\delta$ is nondegenerate as well as in the case when $\delta$ is unimodular.

After dividing by $y^{\alpha^{0}}$ and denoting $\alpha^{j}-\alpha^{0}$ by $\alpha_{j}, j=1, \ldots, N-1$ we can assume that the reduction has the following form

$$
f\left(y_{1}, \ldots, y_{k}\right)=1+\sum_{i=1}^{k} y_{1}^{\alpha_{i 1}} \ldots y_{k}^{\alpha_{i k}}+\sum_{i=1}^{m} z_{i} y_{1}^{\alpha_{k+i, 1}} \ldots y_{k}^{\alpha_{k+i, k}}=0
$$

where the matrix $\delta=\left(\alpha_{i j}\right), i, j=1, \ldots, k$ is nondegenerate. Let $\mathbf{b}_{0}, \mathbf{b}_{1}, \ldots, \mathbf{b}_{k}$ be the first $k+1$ rows of the matrix $B$. In this case we have the following statement.

Theorem 1. The vector-function $y(s)=\left(y_{1}(s), \ldots, y_{k}(s)\right)$ with the coordinates

$$
y_{j}(s)=\prod_{\nu=1}^{k}\left(\frac{\left\langle\mathbf{b}_{\nu}, s\right\rangle}{\left\langle\mathbf{b}_{0}, s\right\rangle}\right)^{\chi_{j \nu}}, j=1,2, \ldots, k
$$

where $\chi_{j \nu}$ are the entries of the matrix $\delta^{-1}$, parameterizes the set of singular points of the reduced hypersurface ( 7$)$.

Proof. Firstly, we consider the case when $\delta$ is the unit matrix, i.e. when $f$ is of the type

$$
f\left(y_{1}, \ldots, y_{k}\right):=1+y_{1}+\ldots+y_{k}+\sum_{i=1}^{m} z_{i} y_{1}^{\alpha_{k+i, 1}} \ldots y_{k}^{\alpha_{k+i, k}}=0
$$

with an associated matrix

$$
A=\left(\begin{array}{ccccccccc}
1 & 1 & 1 & \ldots & 1 & 1 & 1 & \ldots & 1 \\
0 & 1 & 0 & \ldots & 0 & \alpha_{k+1,1} & \alpha_{k+2,1} & \ldots & \alpha_{N-1,1} \\
0 & 0 & 1 & \ldots & 0 & \alpha_{k+1,2} & \alpha_{k+2,2} & \ldots & \alpha_{N-1,2} \\
\ldots & \ldots & \ldots & \ldots & \ldots & \ldots & \ldots & \ldots & \ldots \\
0 & 0 & 0 & \ldots & 1 & \alpha_{k+1, k} & \alpha_{k+2, k} & \ldots & \alpha_{N-1, k}
\end{array}\right)
$$

Choose the dual matrix

$$
\begin{gathered}
B=\left(\begin{array}{c}
b_{0} \\
b_{1} \\
\vdots \\
b_{k} \\
E_{m}
\end{array}\right), \\
-673-
\end{gathered}
$$


where

$$
\begin{aligned}
& b_{0}=\left(-1+\sum_{j=1}^{k} \alpha_{k+1, j} ;-1+\sum_{j=1}^{k} \alpha_{k+2, j} ; \ldots ;-1+\sum_{j=1}^{k} \alpha_{N-1, j}\right) \\
& b_{1}=\left(-\alpha_{k+1,1} ;-\alpha_{k+2,1} ; \ldots ;-\alpha_{N-1,1}\right) \\
& \ldots \\
& b_{k}=\left(-\alpha_{k+1, k} ;-\alpha_{k+2, k} ; \ldots ;-\alpha_{N-1, k}\right) .
\end{aligned}
$$

Due to the Horn-Kapranov formula (6) the discriminantal set of the equation (8) is given by the following parametrization

$$
z_{i}=s_{i}\left\langle b_{0}, s\right\rangle^{-1+\sum_{j=1}^{k} \alpha_{k+i, j}}\left\langle b_{1}, s\right\rangle^{-\alpha_{k+i, 1}} \ldots\left\langle b_{k}, s\right\rangle^{-\alpha_{k+i, k}}, i=1,2 \ldots, m,
$$

where $s=\left(s_{1}, \ldots, s_{m}\right)$.

Lemma 1. The vector-function $y(s)=\left(y_{1}(s), \ldots y_{k}(s)\right)$ with coordinates

$$
y_{1}(s)=\frac{\left\langle b_{1}, s\right\rangle}{\left\langle b_{0}, s\right\rangle}, \ldots, y_{k}(s)=\frac{\left\langle b_{k}, s\right\rangle}{\left\langle b_{0}, s\right\rangle}
$$

satisfies the system of equations

$$
f\left(y_{1}, \ldots, y_{k}\right)=\frac{\partial f\left(y_{1}, \ldots, y_{k}\right)}{\partial y_{1}}=\ldots=\frac{\partial f\left(y_{1}, \ldots, y_{k}\right)}{\partial y_{k}}=0 .
$$

Proof. Let us substitute $y=y(s)$ into the equation (8) with the coefficients $z==(B s)^{B}$. We get the following expression

$$
\begin{gathered}
1+\frac{\left\langle b_{1}, s\right\rangle}{\left\langle b_{0}, s\right\rangle}+\ldots+\frac{\left\langle b_{k}, s\right\rangle}{\left\langle b_{0}, s\right\rangle}+ \\
+\sum_{i=1}^{m}\left(s_{i}\left\langle b_{0}, s\right\rangle^{-1+\sum_{j=1}^{k} \alpha_{k+i, j}}\left\langle b_{1}, s\right\rangle^{-\alpha_{k+i, 1}} \ldots\left\langle b_{k}, s\right\rangle^{-\alpha_{k+i, k}}\right)\left(\frac{\left\langle b_{1}, s\right\rangle}{\left\langle b_{0}, s\right\rangle}\right)^{\alpha_{k+i, 1}} \ldots\left(\frac{\left\langle b_{k}, s\right\rangle}{\left\langle b_{0}, s\right\rangle}\right)^{\alpha_{k+i, k}}= \\
=\frac{\left\langle b_{0}, s\right\rangle+\left\langle b_{1}, s\right\rangle+\ldots+\left\langle b_{k}, s\right\rangle}{\left\langle b_{0}, s\right\rangle}+\frac{s_{1}+\ldots+s_{m}}{\left\langle b_{0}, s\right\rangle} .
\end{gathered}
$$

The last sum vanishes, since

$$
\left\langle b_{0}, s\right\rangle+\left\langle b_{1}, s\right\rangle+\ldots+\left\langle b_{k}, s\right\rangle=-\left(s_{1}+\ldots+s_{m}\right) .
$$

Recall that the sum of all rows of the matrix $B$ is equal to zero, and $B$ consists of rows $b_{0}, \ldots, b_{k}$ supplemented by the unit $m \times m$-matrix. So, $y(s)$ annihilates $f(y)$ when $z=(B s)^{B}$.

Similarly for the derivatives, one has as follows

$$
\frac{\partial g\left(y_{1}(s), \ldots y_{k}(s)\right)}{\partial y_{j}}=1+\frac{1}{\left\langle b_{0}, s\right\rangle} \sum_{i=1}^{m} \alpha_{k+i, j} s_{i}=\frac{1}{\left\langle b_{j}, s\right\rangle}\left(\left\langle b_{j}, s\right\rangle+\sum_{i=1}^{m} \alpha_{k+i, j} s_{i}\right)=0
$$

The last expression vanishes due to the property of vectors $b_{j}$. So, the proof of Lemma 1 is completed.

In order to continue the proof of Theorem 1 let us turn to the equation (7). We introduce the monomial change

$$
x_{i}=y_{1}^{\alpha_{i 1}} \ldots y_{k}^{\alpha_{i k}}, i=1,2, \ldots, k
$$


which can be rewritten in the matrix form as $x=y^{\delta}$. Since $\delta$ is nondegenerate, one has

$$
y=x^{\delta^{-1}} \text {. }
$$

Let us write the matrix $A=\left(\alpha_{i j}\right)$ in the block form $A=\left(\delta, \delta^{\prime}\right)$. Then after the substitution $y=x^{\delta^{-1}}$ in (7) we get

$$
1+\sum_{i=1}^{k} x_{i}+\sum_{i=1}^{m} z_{i}\left(x^{\delta^{-1} \delta^{\prime}}\right)_{i}=0
$$

where $\left(x^{\delta^{-1}} \delta^{\prime}\right)_{i}$ is the $i$-th coordinate of the vector $x^{\delta^{-1} \delta^{\prime}}$. The exponents in equation (11) form the $k \times N$-matrix $\left(E_{k}, \delta^{-1} \delta^{\prime}\right)$. This matrix supplemented by the row of units looks like (9) where the block $\delta^{\prime}$ is changed by $\delta^{-1} \delta^{\prime}$ :

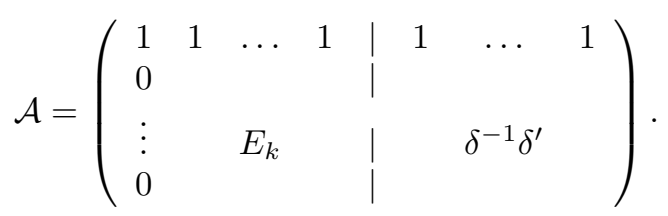

The computation shows that the dual matrix to $\mathrm{A}$ is the matrix

$$
\mathcal{B}=\left(\begin{array}{c}
\mathbf{b}_{0} \\
\mathbf{b}_{1} \\
\vdots \\
\mathbf{b}_{k} \\
E_{m}
\end{array}\right)
$$

Further applying Lemma 1 we complete the proof of Theorem 1.

\section{Rational expression for singular points}

As it follows from the definition of the $A$-discriminantal set, the singular points of the hypersurface which we consider coincide with the restrictions of solutions to the equation (1) on the $A$-discriminantal set, i.e. with $\left.y(a)\right|_{\nabla_{A}}$. For the reduced equation (7) the singular points $y(z)$ are given by

$$
y(z(s))=y\left((B s)^{B}\right) .
$$

However, according to Kapranov's theorem [6] the parametrization $z=(B s)^{B}$ is the inverse of the logarithmic Gauss map

$$
\gamma: \nabla_{A}^{\prime} \rightarrow \mathbb{C P}^{m-1}
$$

of a reduced $A$-discriminantal set $\nabla_{A}^{\prime}$. At the regular points $z \in \operatorname{reg} \nabla_{A}^{\prime}$ this mapping can be written explicitly (see [8])

$$
\gamma: z \rightarrow\left(z_{1}\left(\Delta^{\prime}\right)_{z_{1}}: \ldots: z_{m}\left(\Delta^{\prime}\right)_{z_{m}}\right)=\left(s_{1}: \ldots: s_{m}\right)
$$

where for simplicity we write $\Delta^{\prime}$ instead of $\Delta_{A}^{\prime}$. Therefore, by Theorem 1 we get the following statement.

Theorem 2. The singular points of the reduced hypersurface (7) over the set reg $\nabla_{A}^{\prime}$ admit in global coordinates $z$ the following radical representation:

$$
y_{j}(z)=\prod_{\nu=1}^{k}\left(\frac{\left\langle b_{\nu}, \gamma(z)\right\rangle}{\left\langle b_{0}, \gamma(z)\right\rangle}\right)^{\chi_{j \nu}}, j=1,2, \ldots, k
$$


where $\chi_{j \nu}$ are the entries of the matrix $\delta^{-1}$.

Now we can formulate the main result.

Theorem 3. Let the set $A$ in (1) generate $\mathbb{Z}^{k}$ as a group. The singular points $y(a)$ of the hypersurface (1) over the set reg $\nabla_{A}$ admit a rational representation.

Proof. We consider an arbitrary reduction of the type $(7)$ with fixed coefficients $a_{\alpha^{j_{0}}}=\ldots=$ $=a_{\alpha^{j_{k}}}=1$. Let $B_{J^{\prime}}$ be the submatrix of the dual matrix $B$ consisting of rows $b_{\alpha^{j_{1}}}, \ldots, b_{\alpha^{j_{k}}}$. Then by Theorem 2 the singular points of the reduced hypersurface can be found in the following way:

$$
y(z)=\left(\frac{\left\langle B_{J^{\prime}}, \gamma(z)\right\rangle}{\left\langle b_{j_{0}}, \gamma(z)\right\rangle}\right)^{\alpha_{J}^{-1}}
$$

Consider all subsets $J=\left\{j_{0}, j_{1}, \ldots, j_{k}\right\} \subset\{1, \ldots, N\}$ for which the corresponding matrices $\delta_{J}$ are nondegenerate. Then there exist integer numbers $q_{J}$ such that

$$
\sum_{J} q_{J} \delta_{J}=E_{k}
$$

Consequently, we have

$$
y(a)=y^{E_{k}}=y^{\sum_{J} q_{J} \delta_{J}}=\prod_{J}\left(\frac{\left\langle B_{J^{\prime}}, \gamma(z)\right\rangle}{\left\langle b_{j_{0}}, \gamma(z)\right\rangle}\right)^{\delta_{J}^{-1} q_{J} \delta_{J}}=\prod_{J}\left(\frac{\left\langle B_{J^{\prime}}, \gamma(z)\right\rangle}{\left\langle b_{j_{0}}, \gamma(z)\right\rangle}\right)^{q_{J}} .
$$

The last term is a rational expression in variables $z$. Since by Kapranov's theorem $\gamma$ is a birational map we get rationality of $y(a)$ in variables $a$.

\section{Example}

Let us consider the following polynomial equation

$$
a_{00}+a_{10} y_{1}+a_{01} y_{2}+a_{31} y_{1}^{3} y_{2}+a_{63} y_{1}^{6} y_{2}^{3}=0
$$

It is associated with the matrix

$$
A=\left(\begin{array}{lllll}
1 & 1 & 1 & 1 & 1 \\
0 & 1 & 0 & 3 & 6 \\
0 & 0 & 1 & 1 & 3
\end{array}\right)
$$

which has the right annulator

$$
B=\left(\begin{array}{rr}
3 & 8 \\
-3 & -6 \\
-1 & -3 \\
1 & 0 \\
0 & 1
\end{array}\right)
$$

The reduced equation looks as follows:

$$
f=1+y_{1}+y_{2}+z_{1} y_{1}^{3} y_{2}+z_{2} y_{1}^{6} y_{2}^{3}=0 .
$$


According to (6) the parametrization of the reduced $A$-discriminantal set $\nabla^{\prime}=\nabla_{A}^{\prime}$ for $f$ is

$$
\begin{gathered}
z_{1}=s_{1}\left(3 s_{1}+8 s_{2}\right)^{3}\left(-3 s_{1}-6 s_{2}\right)^{-3}\left(-s_{1}-3 s_{2}\right)^{-1}=\frac{(3+8 s)^{3}}{(3+6 s)^{3}(1+3 s)}, \\
z_{2}=s_{2}\left(3 s_{1}+8 s_{2}\right)^{8}\left(-3 s_{1}-6 s_{2}\right)^{-6}\left(-s_{1}-3 s_{2}\right)^{-3}=-\frac{s(3+8 s)^{8}}{(3+6 s)^{6}(1+3 s)^{3}},
\end{gathered}
$$

where $s:=\frac{s_{2}}{s_{1}}$ is an affine coordinate in $\mathbb{C P}_{1}$. After elimination of the parameter $s$ in the system (14)-(15) we get the reduced $A$-discriminant $\Delta^{\prime}=\Delta_{A}^{\prime}$ :

$$
\begin{aligned}
\Delta^{\prime}=-262144 z_{2}^{3}+ & 331776 z_{1} z_{2}^{3}+331776 z_{1}^{3} z_{2}^{2}-61236 z_{1}^{6} z_{2}-61236 z_{1}^{2} z_{2}^{3}-19683 z_{2}^{4}-398034 z_{1}^{4} z_{2}^{2}+ \\
+ & 59049 z_{1}^{7} z_{2}+19683 z_{1}^{3} z_{2}^{3}+59049 z_{1}^{5} z_{2}^{2}-19683 z_{1}^{8}+19683 z_{1}^{9} .
\end{aligned}
$$

The matrix $\delta$ for the equation (12) is the unit matrix, therefore by (12) we get the following formulas for singular points:

$$
y_{1}=\frac{-3 z_{1}\left(\Delta^{\prime}\right)_{z_{1}}-6 z_{2}\left(\Delta^{\prime}\right)_{z_{2}}}{3 z_{1}\left(\Delta^{\prime}\right) z_{1}+8 z_{2}\left(\Delta^{\prime}\right) z_{2}}, \quad y_{2}=\frac{-z_{1}\left(\Delta^{\prime}\right)_{z_{1}}-3 z_{2}\left(\Delta^{\prime}\right) z_{2}}{3 z_{1}\left(\Delta^{\prime}\right)_{z_{1}}+8 z_{2}\left(\Delta^{\prime}\right)_{z_{2}}}
$$

The derivatives

$$
\frac{d z_{1}}{d s}=-\frac{(3+8 s)^{2}(4 s+1)^{2}}{9(1+2 s)^{4}(1+3 s)^{2}}, \quad \frac{d z_{2}}{d s}=-\frac{(3+8 s)^{7}(4 s+1)^{2}}{243(1+2 s)^{7}(1+3 s)^{4}}
$$

vanish when $s=-\frac{1}{4}$ and $s=-\frac{3}{8}$. It means that $\nabla_{A}$ has two singular points

$$
z\left(-\frac{1}{4}\right)=\left(\frac{32}{27}, \frac{1024}{729}\right) \text { and } z\left(-\frac{3}{8}\right)=(0,0) .
$$

Elimination of the parameter $s$ in the system (14)-(15) leads us to the $A$-discriminant $\Delta^{\prime}=\Delta_{A}^{\prime}$ :

$$
\begin{aligned}
\Delta^{\prime}=-262144 z_{2}^{3}+ & 331776 z_{1} z_{2}^{3}+331776 z_{1}^{3} z_{2}^{2}-61236 z_{1}^{6} z_{2}-61236 z_{1}^{2} z_{2}^{3}-19683 z_{2}^{4}-398034 z_{1}^{4} z_{2}^{2}+ \\
& +59049 z_{1}^{7} z_{2}+19683 z_{1}^{3} z_{2}^{3}+59049 z_{1}^{5} z_{2}^{2}-19683 z_{1}^{8}+19683 z_{1}^{9}
\end{aligned}
$$

Consider the Taylor decomposition of $\Delta^{\prime}$ at the point $z\left(-\frac{1}{4}\right)$, i.e. by powers of $p=z_{1}-\frac{32}{27}$ and $q=z_{2}-\frac{1024}{729}$

$$
\Delta^{\prime}=\frac{68719476736}{19683} p^{3}-\frac{536870912}{243} p^{2} q+\frac{4194304}{9} p q^{2}-32768 q^{3}+\frac{486539264}{81} p^{4}+r(p, q),
$$

where $r(p, q)$ is a sum of monomials of degree $\geqslant 4$ except the monomial $p^{4}$. Here the initial homogeneous cubic form is a cube power of an affine polynomial

$$
\frac{32768}{14348907}\left(1152 z_{1}-243 z_{2}-1024\right)^{3}
$$

Consequently, in coordinates $m=1152 z_{1}-243 z_{2}-1024$ and $l=z_{1}-\frac{32}{27}$ the discriminant has the form

$$
\Delta^{\prime}=a m^{3}+b l^{4}+\ldots, \quad a \neq 0, \quad b \neq 0
$$


It means that $z=(32 / 27,1024 / 729)$ is a cuspidal point of the type $(4,3)$ for the discriminant $\Delta^{\prime}$.

Now we have to study singular types of singular points of the complex curve (13) which are given by Theorem 2:

$$
y_{1}(s)=\frac{-3-6 s}{3+8 s}, \quad y_{2}(s)=\frac{-1-3 s}{3+8 s} .
$$

At the singular points $y(s)$ we have the following expression for the Hessian of $f$ :

$$
\frac{\partial^{2} f}{\partial y_{1}^{2}} \frac{\partial^{2} f}{\partial y_{2}^{2}}-\left(\frac{\partial^{2} f}{\partial y_{1} \partial y_{2}}\right)^{2}=-\frac{(3+8 s)^{2}(1+4 s)^{2}}{(1+2 s)^{2}(1+3 s)^{2}}
$$

Therefore, only $y\left(-\frac{1}{4}\right)=\left(-\frac{3}{2},-\frac{1}{4}\right)$ is not a Morse point.

Consider the expression of the polynomial (13) at the point $y\left(-\frac{1}{4}\right)$ :

$$
\begin{gathered}
f=-12\left(y_{2}+1 / 4\right)^{2}-4\left(y_{1}+3 / 2\right)\left(y_{2}+1 / 4\right)-\frac{1}{3}\left(y_{1}+3 / 2\right)^{2}+16\left(y_{2}+1 / 4\right)^{3}+ \\
+48\left(y_{1}+3 / 2\right)\left(y_{2}+1 / 4\right)^{2}+\frac{44}{3}\left(y_{1}+3 / 2\right)^{2}\left(y_{2}+1 / 4\right)+32 / 27\left(y_{1}+3 / 2\right)^{3}-\frac{448}{27}\left(y_{1}+3 / 2\right)^{3}\left(y_{2}+1 / 4\right)- \\
-64\left(y_{1}+3 / 2\right)\left(y_{2}+1 / 4\right)^{3}-80\left(y_{1}+3 / 2\right)^{2}\left(y_{2}+1 / 4\right)^{2}-\frac{20}{27}\left(y_{1}+3 / 2\right)^{4}+\frac{320}{3}\left(y_{1}+3 / 2\right)^{2}\left(y_{2}+1 / 4\right)^{3}+ \\
+\frac{640}{9}\left(y_{1}+3 / 2\right)^{3}\left(y_{2}+1 / 4\right)^{2}+\frac{16}{81}\left(y_{1}+3 / 2\right)^{5}+\frac{80}{9}\left(y_{1}+3 / 2\right)^{4}\left(y_{2}+1 / 4\right)+\ldots
\end{gathered}
$$

After the change of variables

$$
y_{1}+\frac{3}{2}=-\frac{u}{15}+\frac{v}{30}, \quad y_{2}+\frac{1}{4}=\frac{8 u}{45}-\frac{v}{180}
$$

we get

$$
f=\frac{1}{3} u^{2}+\frac{1}{8201250} v^{4}+r(u, v)
$$

where $r(u, v)$ consists of monomials of weighted degree $\geqslant 4$ with respect to the weight $(2,1)$. This means that $y\left(-\frac{1}{4}\right)$ is a self-intersection point for the curve (13) with a common tangent line.

The first two authors were supported by the grant of Ministry of Education and Science of the Russian Federation (no. 1.2604.2017/PCh). The third author was supported by the grant of the Russian Federation Government for scientific research under the supervision of leading scientists at Siberian Federal University (no. 14.Y26.31.0006) and grant RFBR, no. 18-51-41011 Uzb

\section{References}

[1] I.Gelfand, M.Kapranov, A.Zelevinsky, Discriminants, resultants and multidimensional determinants, Birkhäuser: Boston, 1994.

[2] I.A.Antipova, A.K.Tsikh, The discriminant locus of a sistem of $n$ Laurent polynomials in $n$ variables, Izv. Math., 76(2012), no 5, 881-906.

[3] E.N.Mikhalkin, A.K.Tsikh, Singular strata of cuspidal type for the classical discriminant, $S b$. Math., 206(2015), no 2, 282-310.

[4] M.Passare, A.Tsikh, Algebraic equations and hypergeometric series, The legacy of Niels Henrik Abel, Springer, Berlin-Heidelberg-New York, 2004, 653-672. 
[5] I.A.Antipova, E.N.Mikhalkin, A.K.Tsikh, Rational expressions for multiple roots of algebraic equations, Matem sb, 209(2018), no 10, 3-30 (in Russian). Engl. transl. in Sb. Math., 209(92018), in press.

[6] M.M.Kapranov, A characterization of A-discriminantal hypersurfaces in terms of the logarithmic Gauss map, Math. Ann, 290(1991), 277-285.

[7] H.Lee, B.Sturmfels, Duality of Multiple Root Loci, Journal of Algebra, 446(2016), 499-526.

[8] G.Mikhalkin, Real algebraic curves, the moment map and amoebas, Ann. of Math., 151(2000), no. 2, 309-326.

\section{Сингулярные точки комплексных алгебраических гиперповерхностей}

Ирина А. Антипова

Институт космических и информационных технологий

Сибирский федеральный университет

Киренского, 26, Красноярск, 660074

Россия

\section{Евгений Н. Михалкин}

Август К. Цих

Институт математики и фундаментальной информатики

Сибирский федеральный университет

Свободный, 79, Красноярск, 660041

Россия

$\overline{\text { Рассматривается комплексная гиперповерхность } V \text {, заданная алгебраическим уравнением } c k}$ неизвестными и с переменными коэффициентами, причем множество $A \subset \mathbb{Z}^{k}$ показателей мономов уравнения произвольное, но фиксированное. Таким образом, мы рассматриваем семейство гиперповерхностей, параметризованных наборами коэффициентов $a=\left(a_{\alpha}\right)_{\alpha \in A} \in \mathbb{C}^{A}$. Доказывается, что если $A$ порождает решетку $\mathbb{Z}^{k}$ как группу, то над множеством регулярных точек $A$-дискриминантного множества сингулярные точки гиперповерхности $V$ рационально выражаются через коэффициенты а.

Ключевые слова: особая точка, А-дискриминант, логарифмическое отображение Гаусса. 